\title{
Potentiation of mitotane action by rosuvastatin: New insights for adrenocortical carcinoma management
}

\author{
GEOFFREY BOULATE ${ }^{1,2}$, LARBI AMAZIT ${ }^{1-3}$, ANNABELLE NAMAN $^{1,2}$, ATMANE SECK $^{4}$, ANGELO PACI $^{4}$, \\ ANNE LOMBES ${ }^{5}$, ERIC PUSSARD ${ }^{6}$, ERIC BAUDIN ${ }^{1,2,7}$, MARC LOMBES $^{1,2^{*}}$ and SÉGOLÈNE HESCOT ${ }^{1,2,8^{*}}$ \\ ${ }^{1}$ INSERM UMR-S 1185, 94276 Le Kremlin-Bicêtre Cedex; ${ }^{2}$ Paris Saclay University, 94270 Le Kremlin-Bicêtre Cedex; \\ ${ }^{3}$ Biomedical Institute of Bicêtre, UMS-32, 94276 Le Kremlin-Bicêtre Cedex; ${ }^{4}$ Department of Pharmacology, \\ Gustave Roussy, 94800 Villejuif; ${ }^{5}$ INSERM UMRS 1016, Cochin Institute, 75014 Paris; \\ ${ }^{6}$ Department of Genetics and Hormonology, APHP, 94270 Le Kremlin-Bicêtre Cedex; \\ ${ }^{7}$ Department of Endocrine Oncology, Gustave Roussy, 94800 Villejuif; \\ ${ }^{8}$ Department of Nuclear Medicine, Curie Institute, 75248 Paris, France
}

Received November 28, 2018; Accepted March 15, 2019

DOI: 10.3892/ijo.2019.4770

\begin{abstract}
Mitotane(alsotermed $o, p^{\prime}-D D D$ ) is the mosteffective therapy for advanced adrenocortical carcinoma (ACC). Mitotane-induced dyslipidemia is treated with statins. Mitotane and statins are known to exert anti-proliferative effects in vitro; however, the effects of statins have never been directly evaluated in patients with ACC and ACC cells, at least to the best of our knowledge. Thus, in this study, we aimed to examine the effects of the rosuvastatin on ACC cells. It has been shown that the combined use of mitotane and statins significantly increases the tumor control rate in patients with ACC; however, it would be of interest to elucidate the molecular mechanisms involved in this potentiation. In this study, we examined the effects of mitotane, rosuvastatin and their combination in NCI-H295R human ACC cells using proliferation assays, gene expression analyses and free intracellular cholesterol measurements. The results revealed that mitotane dose-dependently reduced cell viability, induced apoptosis and increased intracellular free cholesterol levels, considered as one of the key features of mitotane action, while rosuvastatin alone reduced cell viability and increased apoptosis at high concentrations. We also demonstrated that rosuvastatin potentiated the effects of mitotane by reducing cell viability, inducing apoptosis, increasing intracellular free cholesterol levels, and by decreasing the expression
\end{abstract}

Correspondence to: Dr Marc Lombes, INSERM UMR-S 1185 , Fac Med Paris Sud, 63 Rue Gabriel Péri, 94276 Le Kremlin-Bicêtre Cedex, France

E-mail:marc.lombes@u-psud.fr

${ }^{*}$ Contributed equally

Key words: adrenocortical carcinoma, mitotane, statins, rosuvastatin, H295R cells of 3-hydroxy-3-methylglutaryl-CoA reductase (HMGCR) and ATP binding cassette subfamily a member 1 (ABCAl), genes involved in cholesterol metabolism, and inhibiting steroidogenesis. Collectively, potentiating the effects of mitotane with the use of rosuvastatin may provide novel therapeutic strategies for ACC, given that the combination of these drugs, pending clinical validation, may lead to the better management of ACC.

\section{Introduction}

Mitotane (also termed $o, p^{\prime}-D D D$ ) is the only drug approved for the treatment of metastatic adrenocortical carcinoma (ACC) (1); however, its molecular mechanisms of action remain to be fully elucidated. The recommended therapeutic window of plasma mitotane levels in patients is between 14 and $20 \mathrm{mg} / \mathrm{l}$, corresponding approximately to $50 \mu \mathrm{M}$ (1). We previously reported that mitotane induces mitochondrial dysfunction in NCI-H295R human adrenocortical cells, including respiratory chain inhibition and mitochondrial fragmentation (2). Moreover, a mitochondrial uptake of mitotane leading to cell apoptosis has been shown (3). We have also previously demonstrated that mitotane disrupts the integrity of mitochondrial-associated membranes (MAMs) using metabolomic, lipidomic and imaging approaches (4). Indeed, MAMs constitute pivotal intracellular structures controlling key cellular processes, such as apoptosis, calcium homeostasis, phospholipid metabolism, mitochondrial function, cholesterol metabolism and steroid synthesis, notably in adrenocortical cells. Recently, sterol-O-acyl transferase 1 (SOAT1), the enzyme that metabolizes free cholesterol to cholesterol esters, was also proposed as a new potential target of mitotane (5). Accordingly, Sbiera et al (5) hypothesized that mitotane could induce endoplasmic reticulum (ER) stress, through SOAT1 inhibition, leading to increased intracellular free cholesterol concentrations followed by apoptosis. In steroidogenic cells, such as adrenocortical cells, cholesterol metabolism plays a major role since cholesterol is the main precursor for steroid 
biosynthesis. There are at least 4 sources of free cholesterol in the adrenocortical cell: An exogenous source of cholesteryl esters (CEs) originating from: i) low-density lipoprotein (LDL) through low-density lipoprotein receptor (LDL-R); and ii) high-density lipoprotein (HDL) through scavenger receptor $\mathrm{B}$ (SrB1); iii) lipid droplets; and iv) de novo cholesterol synthesis through 3-hydroxy-3-methyl-glutaryl-coenzyme A (HMGCoA) reductase (HMGCR) activity, also known as the mevalonate pathway. Free cholesterol may then be transported by steroidogenic acute regulatory protein (StAR) to MAMs to be converted in pregnenolone by cytochrome P450, family 11 , subfamily A, polypeptide 1 (CYP11A1) whereas an efflux of free cholesterol may also occur through ATP-binding cassette transporter (ABCA1). Mitotane is a lipophilic molecule that circulates either free or is bound to lipoproteins. Furthermore, mitotane induces dyslipidemia with increased LDL, HDL and triglycerides concentrations (6). This dyslipidemia strikingly reduces mitotane efficacy in vitro as demonstrated by the higher anti-proliferative and pro-apoptotic effects of mitotane when NCI-H295R cells are cultured in lipoprotein-free medium (3). Moreover, this dyslipidemia leads to an overestimation of plasma mitotane levels in patients (7), and is generally treated by statins. Lastly, in a retrospective study of 26 patients with ACC (3), the combination of mitotane and statins was shown to be significantly associated with a better tumor control according to Response Evaluation Criteria In Solid Tumors criteria (RECIST) (8). Thus, the mechanisms through which statins may potentiate the effects of mitotane are therefore considered of relevance for investigation.

Statins inhibit HMGCR and exert an anti-proliferative effect in vitro on several cancer cell lines, such as lung, prostate, breast, ovary, leukemia and myeloma cells (9). These effects could be linked to an inhibition of the mevalonate pathway (10); however, they have never been investigated in adrenocortical cells to date, at least to the best of our knowledge.

The aim of the present study was to evaluate the effects of mitotane alone or in association with statins in NCI-H295R human ACC cells.

\section{Materials and methods}

Human adrenocortical cell culture. For in vitro experiments, NCI-H295R (hereon referred to as H295R) human ACC cells (from passage 7 to 12) obtained from Gustave Roussy, Universite Paris Sud, Villejuif, France and used in our previous studies (2-4,11), were cultured as previously described (3). The H295R cells were cultured in DMEM/HAM'S F-12 (PAA, Les Mureaux, France) supplemented with 20 mM HEPES (Invitrogen, Life Technologies/Thermo Fisher Scientific, Waltham, MA, USA), antibiotics (penicillin $100 \mathrm{IU} / \mathrm{ml}$ and streptomycin $100 \mu \mathrm{g} / \mathrm{ml}$ ) and $2 \mathrm{mM}$ glutamine. The medium for $\mathrm{H} 295 \mathrm{R}$ cell culture was enriched with $10 \%$ fetal bovine serum and a mixture of insulin/transferrin/selenium. The cells were cultured at $37^{\circ} \mathrm{C}$ in a humidified incubator with $5 \% \mathrm{CO}_{2} . o, p^{\prime}-D D D$ (Sigma-Aldrich, St. Louis, MO, USA), and rosuvastatin (Sigma-Aldrich) were solubilized in dimethyl sulfoxide (DMSO; Sigma-Aldrich) and used at the indicated concentrations ranging from 0 to $100 \mu \mathrm{M}$. In all experiments, the percentage of DMSO in the culture medium never exceeded $0.1 \% \mathrm{v} / \mathrm{v}$. Given that $o, p^{\prime}-D D D$ induces hepatic
CYP3A4 activity (12), we selected rosuvastatin for use in our experiments, a statin not metabolized by CYP3A4.

Cell viability and apoptosis analysis. Cell viability assays were performed using WST1 assay (Roche, Basel, Switzerland) and apoptosis tests were performed using the Caspase-Glo 3/7 assay (Promega, Madison, WI, USA) according to the manufacturer's recommendations. The cells were cultured in 96-well plates and treated with $0-100 \mu \mathrm{M}$ mitotane alone or with rosuvastatin for various periods of time (0 to $72 \mathrm{~h})$. The number of cells per well was 3 to $10 \times 10^{3}$. Optical densities were measured $4 \mathrm{~h}$ after the addition of WST1 solution $(10 \mu \mathrm{l}$ per well) by spectrophotometry at $450 \mathrm{~nm}$ (Viktor multilabel plate reader; PerkinElmer, Waltham, MA, USA). The results were validated by cell counting with the cell counter method (TC20 automated cell counter; Bio-Rad Laboratories, Hercules, CA, USA). Luminescence was measured $1 \mathrm{~h}$ after the addition of Caspase-Glo 3/7 solution (equal volume) by luminometry (Viktor multilabel plate reader; PerkinElmer).

Western blot analysis. Total protein extracts were prepared and western blot analyses were performed as previously described (11). Total protein extracts were prepared from cells lysed in lysis buffer (50 mM Tris- $\mathrm{HCl}, \mathrm{pH} 7.5$, $150 \mathrm{mM} \mathrm{NaCl}, 5 \mathrm{mM}$ EDTA, $30 \mathrm{mM}$ Na pyrophosphate, $50 \mathrm{mM} \mathrm{Na}$ fluoride and $1 \%$ Triton X-100) and $1 \mathrm{X}$ protease inhibitor (Sigma-Aldrich), $40 \mu \mathrm{g}$ proteins were loaded by lane. Following protein blotting on an Odyssey nitrocellulose membrane (LI-COR, Lincoln, NE, USA), the blots were incubated for $1 \mathrm{~h}$ at room temperature in a blocking buffer [5\% fat-free milk in phosphate-buffered saline (PBS) with $0.1 \%$ Tween-20] before an overnight incubation at $4^{\circ} \mathrm{C}$. Primary antibodies were a rabbit polyclonal anti-poly(ADP-ribose) polymerase (PARP) antibody (dilution, 1:200; \#9542; Cell Signaling Technology, Danvers, MA, USA). The antibody detected total PARP (116 kDa) and cleaved PARP $(89 \mathrm{kDa})$, the ratio of which reflects the pro-apoptotic status. The normalizing antibody was the anti- $\alpha$-tubulin (dilution 1:1,000; AB_10013740; Sigma-Aldrich). Secondary antibodies were goat anti-mouse IgG $(\mathrm{H}+\mathrm{L})$ cross adsorbed secondary antibody (DyLight 680 conjugated, AB_614942) and goat anti-rabbit IgG $(\mathrm{H}+\mathrm{L})$ DyLight 800 conjugated (dilution 1:10,000, AB_614947) (both from Thermo Fisher Scientific).

Antibodies were diluted in PBS $0.1 \%$ Tween-20 buffer $5 \%$ non-fat milk and added to the membranes for $1 \mathrm{~h}$ at room temperature or overnight at $4^{\circ} \mathrm{C}$, followed by incubation with the indicated secondary antibody for $1 \mathrm{~h}$ at room temperature. Target proteins were detected using Odyssey Fc, Dual-Mode Western Imaging (LI-COR) by fluorescence $(680 \mathrm{~nm}$ wavelength for anti-mouse antibody and $800 \mathrm{~nm}$ for anti-rabbit antibody) and quantified using the Odyssey Fc Dual-mode Western Imaging apparatus from LI-COR as indicated.

Intracellularfree cholesterolmeasurement. The concentrations of free intracellular cholesterol were measured using the Cholesterol Quantification kit (Sigma-Aldrich) according to the manufacturer's recommendations. The cells were cultured in F12 plates (4 wells per condition) treated with mitotane and/or rosuvastatin for $24 \mathrm{~h}$. In these experiments, we used 
serum-free culture media to exclude exogenous cholesterol uptake. The absorbance was read at $570 \mathrm{~nm} 1 \mathrm{~h}$ after the addition of $2 \mu \mathrm{l}$ of cholesterol probe and $2 \mu \mathrm{l}$ of cholesterol enzyme mixture per well. Cholesterol concentrations were calculated according to the established standard curve and normalized to the initial cell count.

Measurement of HMGCR activity. The measurement of HMGCR was carried out using HMGCoA Reductase Assay (Sigma-Aldrich), according to the manufacturer's recommendations. Incubation medium included $\mathrm{HMGCoA}$ (enzyme substrate), NADPH (reduced nicotinamide adenine dinucleotide phosphate), buffer solution and HMG-CoA reductase (provided in the kit). Specific absorbance at $340 \mathrm{~nm}$ was compared in the presence or in the absence of pravastatin, a potent HMGCR activity inhibitor. HMGCR activity was determined by the difference in absorbance slope between these two conditions.

Reverse transcription-quantitative PCR (RT-qPCR). Total RNA was extracted from the H295R cells with the RNeasy kit (Qiagen) according to the manufacturer's recommendations. A total of $1 \mu \mathrm{g}$ total RNAwere subjected to DNase I treatment (Invitrogen/Thermo Fisher Scientific) and reverse-transcribed with 200 units of reverse transcriptase (Superscript II, Invitrogen/Thermo Fisher Scientific). PCR was performed with $100 \mathrm{ng}$ cDNA in the presence of $\mathrm{qPCR}^{\mathrm{TM}}$ Mastermix Plus for Sybr ${ }^{\mathrm{TM}}$-Green I (Eurogentec, Seraing, Belgium) containing $300 \mathrm{nM}$ of specific primers (Table SI). qPCR was carried on an ABI Step One Plus (Applied Biosystems, Foster City, CA, USA) whose parameters were as follows: A pre-cycle at $95^{\circ} \mathrm{C}$ for $20 \mathrm{sec}$ then 40 cycles at $95^{\circ} \mathrm{C}$ for $1 \mathrm{sec}$ followed by 40 cycles at $60^{\circ} \mathrm{C}$ for $20 \mathrm{sec}$. The amount of cytochrome $c$ oxidase subunit II COX 2 transcript in the samples was determined by comparison with the standard range and related to the amount of the $18 \mathrm{~S}$ gene of nuclear origin. For standards preparation, amplicons were subcloned into pGEMT-easy plasmid (Promega) and sequenced to confirm the identity of each sequence. Standard curves were generated using serial dilutions of linearized standard plasmids. Samples were amplified in duplicate or triplicate. Ribosomal $18 \mathrm{~S}$ was used as an internal control for data normalization. qPCR was performed using the Fast SYBR Green Master Mix (Life Technologies/Thermo Fisher Scientific) and carried out on a QuantStudio 6 Flex (Life Technologies/Thermo Fisher Scientific). The relative expression of each gene was expressed as the ratio of attomoles of specific gene to attomoles of $36 B 4 \mathrm{mRNA}$ or femtomoles of $18 S$ rRNA.

Steroidogenesis. Steroid measurements (progesterone, 17OHP) were assayed in the cell supernatants under various conditions after 48-h treatment, by means of liquid chromatography (LC)-mass spectrometry (MS)/MS analysis. LC-MS/MS was performed using a Waters Xevo TQS triple-quadrupole mass spectrometer connected to a Waters Acquity UPLC H-class (Waters SAS, Saint Quentin Yvelines, France). Chromatographic separation was performed on a BEH C18 column $(1.7 \mu \mathrm{m}, 100 \times 2.1)$ at a flow rate of $0.3 \mathrm{ml} / \mathrm{min}$ at $40^{\circ} \mathrm{C}$. The mobile phase consisting of methanol and $5 \mathrm{mmol} / \mathrm{l}$ ammonium formate in water was delivered according to the following gradient: $35 \%$ methanol from 0 to $1.5 \mathrm{~min}$, linear increase to $45 \%$ methanol $(1.5-3 \mathrm{~min})$, then to $63 \%$ methanol (3-7 min) followed by $100 \%$ methanol (7-9 min). Following column washing with $100 \%$ methanol (9-11.5 min), the gradient was reversed to reach initial conditions at $14 \mathrm{~min}$. The injection volume was $10 \mu \mathrm{l}$ and the sample manager was maintained at $10^{\circ} \mathrm{C}$. Detection was performed on a Xevo TQS tandem mass spectrometer (Waters, Paris, France). Instrument optimization for the analytes was conducted by infusing standard solution $(100 \mathrm{pg} / \mathrm{ml})$ of the analytes by the built-in syringe pump at a flow rate of $10 \mu \mathrm{l} / \mathrm{min}$. The following optimized operating conditions were used for the multiple reaction monitoring mode: Capillary voltage, $3.5 \mathrm{kV}$; cone voltage, 4-60 V; collision energy, 15-34 eV; dwell time, 0.03-0.1 sec, depending on the steroid. The mass spectrometer parameters were configured as follows: Desolvation temperature, $500^{\circ} \mathrm{C}$; desolvation nitrogen flow, $790 \mathrm{l} / \mathrm{h}$; source temperature, $150^{\circ} \mathrm{C}$; cone nitrogen flow, $145 \mathrm{l} / \mathrm{h}$. Argon was used as collision gas with a flow rate of $0.14 \mathrm{ml} / \mathrm{min}$. Two mass transitions were monitored for each steroid. System control and data acquisition were achieved with the MassLynx 4.0 software (Waters). Cells were cultured in F6 plates (3 wells per condition) treated with $25 \mu \mathrm{M}$ mitotane (M25) and/or $50 \mu \mathrm{M}$ rosuvastatin (R50) for $48 \mathrm{~h}$.

Statistical analysis. Results are expressed as the means \pm SEM of $n$ independent replicates performed in the same experiment or from separate experiments (n). The non-parametric Mann-Whitney U test was used when appropriate and differences between groups were analyzed using non-parametric Kruskall-Wallis multiple comparison tests followed by a post hoc Dunn's test (Prism software, GraphPad, CA, USA). A P-value of 0.05 was considered to indicate a statistically significant difference.

\section{Results}

Effect of mitotane and rosuvastatin on ACC cell viability and apoptosis. First, we examined the effects of mitotane or rosuvastatin alone on cell viability at the concentration to $100 \mu \mathrm{M}$ and at different time periods (up to $48 \mathrm{~h}$ ). Mitotane $(50 \mu \mathrm{M}$; M50) (Fig. 1A) reduced the absorbance in a time-dependent manner, confirming its anti-proliferative effect. Rosuvastatin alone also induced a time-dependent inhibition of cell viability, providing support for a specific effect of rosuvastatin. This effect, however, was only observed at high concentrations starting from $50 \mu \mathrm{M}$ (R50) (Fig. S1). The combination of mitotane (50 $\mu \mathrm{M}$; M50) and rosuvastatin $(100 \mu \mathrm{M} ; \mathrm{R} 100)$ potentiated the inhibition of cell viability at $48 \mathrm{~h}$ (Fig. 1A), while rosuvastatin $(100 \mu \mathrm{M})$ had no additive effect on the anti-proliferative effects of mitotane at $72 \mathrm{~h}$ (Fig. S2).

Using similar experimental conditions, we then analyzed the index of H295R cell apoptosis using caspase-3/7 activity (Fig. 1B) and cleaved PARP expression (Fig. 1C and D). As expected, mitotane (50 $\mu \mathrm{M}$; M50) alone induced a significant increase in caspase-3/7 activity, while rosuvastatin (100 $\mu \mathrm{M}$; R100) alone had no effect. However, the combination of mitotane and rosuvastatin led to an over-induction of caspase-3/7 activity. Moreover, similar potentiation effects between mitotane and rosuvastatin were observed when 


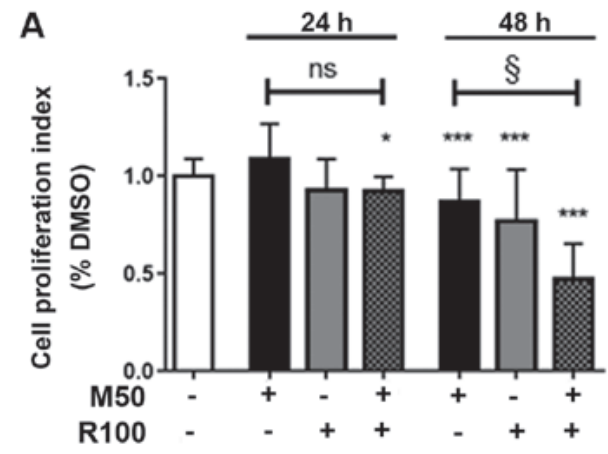

B

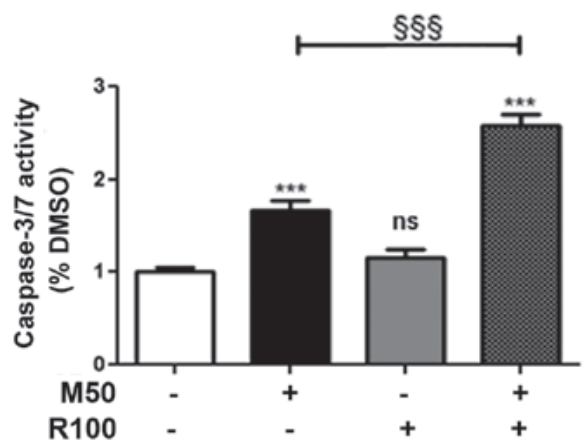

C
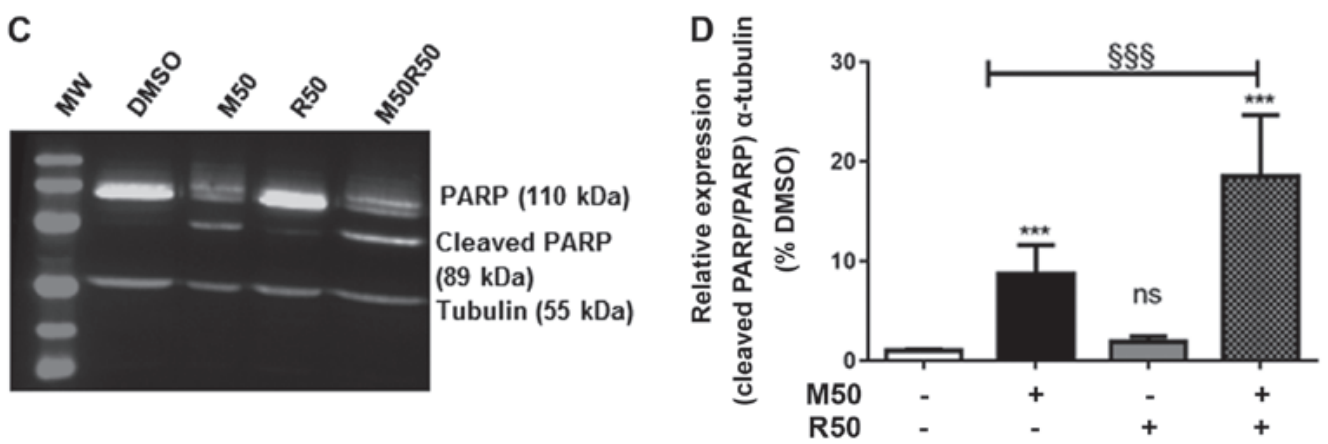

Figure 1. Viability and apoptosis of $\mathrm{H} 295 \mathrm{R}$ cells treated with mitotane and/or rosuvastatin. (A) Cell viability in mitotane and/or rosuvastatin-treated H295R cells. H295R cells were treated for 24 and $48 \mathrm{~h}$ with mitotane $(50 \mu \mathrm{M} ; \mathrm{M} 50)$, rosuvastatin (100 $\mu \mathrm{M} ; \mathrm{R} 100)$, alone or in combination. Cell viability was measured by WST1 assay. Results are the means \pm SEM of 8 to 32 independent determinations from 3 different experiments. (B) Activity of caspase-3/7 in H295R cells treated with mitotane and/or rosuvastatin. H295R cells were treated for $24 \mathrm{~h}$ with mitotane $(50 \mu \mathrm{M} ; \mathrm{M} 50)$, rosuvastatin (100 $\mu \mathrm{M}$; R100), alone or in combination. Apoptotic index was measured using the Caspase-Glo 3/7 kit by luminometry. Results are the means \pm SEM to 16 independent determinations from 2 different experiments. (C) Expression of PARP in mitotane and/or rosuvastatin-treated H295R cells. H295R cells were treated for $24 \mathrm{~h}$ with mitotane (50 $\mu \mathrm{M} ; \mathrm{M} 50)$, rosuvastatin (50 $\mu \mathrm{M} ; \mathrm{R} 50)$, alone or in combination (M50 R50). Steady state levels of PARP and tubulin expression were analyzed by western blot analysis. Representative blots are shown. (D) Expression of PARP in mitotane and/or rosuvastatin-treated H295R cells shown by quantification of band intensities. Ratio between total PARP $(110 \mathrm{kDa})$ and cleaved PARP $(89 \mathrm{kDa})$ normalized to loading controls (tubulin, $55 \mathrm{kDa})$ are shown. Results are the means \pm SEM of 7 to 18 independent determinations from 4 different experiments. Statistical analysis was carried out with the non-parametric Kruskal-Wallis multiple comparison tests followed by a Dunn's post hoc test. ${ }^{*} \mathrm{P}<0.05$ and ${ }^{* * * *} \mathrm{P}<0.001$ vs. DMSO condition; ${ }^{\circledR} \mathrm{P}<0.05$ and ${ }^{\$ \$}{ }^{\$} \mathrm{P}<0.001$ vs. mitotane condition; ns, not significant.

examining the expression of cleaved PARP (Fig. 1C and D), confirming the induction of H295R cell apoptosis when both molecules were used in combination (M50 and R50). As the effects of rosuvastatin were observed at $50 \mu \mathrm{M}$, this concentration was considered as the most relevant for use in our experiments.

Effect of mitotane and rosuvastatin on the intracellular free cholesterol concentration. Since statins and mitotane alter intracellular lipid metabolism $(9,13)$, in this study, we examined the effects of mitotane, rosuvastatin and their association on the level of intracellular free cholesterol, the precursor of steroidogenesis. As shown in Fig. 2, we confirmed that mitotane $(50 \mu \mathrm{M} ; \mathrm{M} 50)$ for $24 \mathrm{~h}$ significantly increased the concentration of intracellular free cholesterol, whereas rosuvastatin $(50 \mu \mathrm{M} ; \mathrm{R} 50)$ alone had no effect. However, no potentiating effect on the free intracellular cholesterol concentration was observed when the cells were exposed to both mitotane and rosuvastatin.

Mitotane does not alter HMGCR activity. Since mitotane increases the intracellular free cholesterol concentrations, we then sought to examine the hypothesis that mitotane may directly increase the activity of HMGCR and may thereby stimulate the mevalonate pathway.

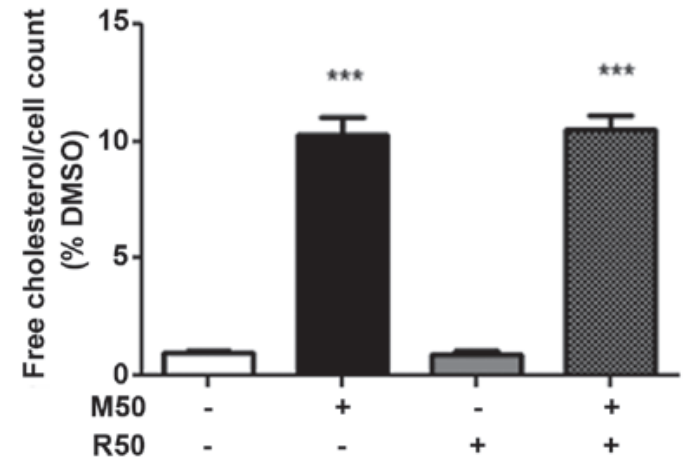

Figure 2. Intracellular free cholesterol concentrations in H295R cells treated with mitotane and/or rosuvastatin. H295R cells were treated for $24 \mathrm{~h}$ alone or in combination with mitotane (50 $\mu \mathrm{M} ; \mathrm{M} 50)$, rosuvastatin $(50 \mu \mathrm{M} ; \mathrm{R} 50)$. Intracellular free cholesterol concentrations were measured using the cholesterol quantification kit as described in the Materials and methods. Results, normalized to the cell number determined in parallel experiments with the WST1 assays, are expressed as the means \pm SEM to 13 of 22 independent determinations from 2 different experiments. Statistical analysis was carried out with the non-parametric Kruskal-Wallis multiple comparison tests followed by a Dunn's post hoc test. ${ }^{* * *} \mathrm{P}<0.0001$ vs. control condition (DMSO).

HMGCR activity was measured at $1,815 \mathrm{U} / \mathrm{mg}$ protein under control conditions and at $1,876 \mathrm{U} / \mathrm{mg}$ following the addition of up to $100 \mu \mathrm{M}$ mitotane, indicating that mitotane exerts 
A

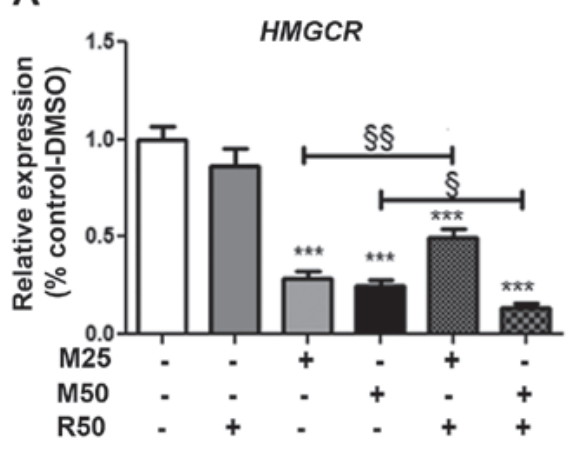

C

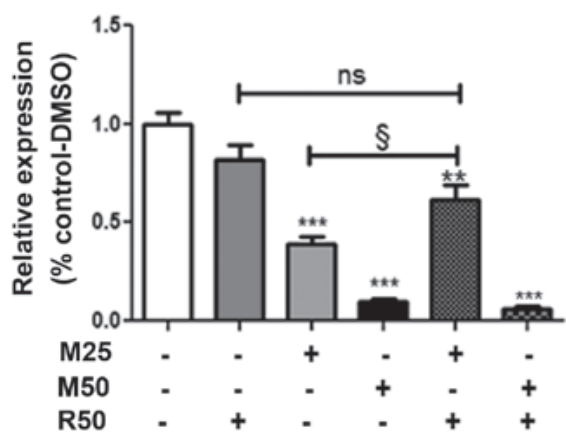

B

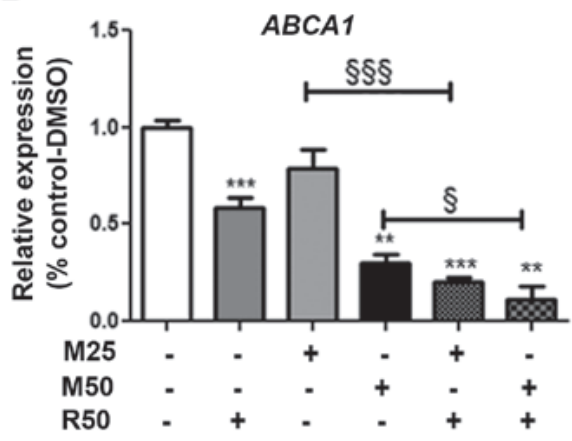

D

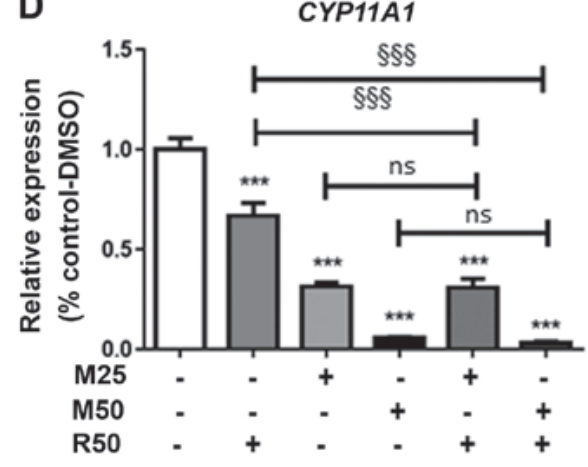

Figure 3. Expression of genes involved in cholesterol metabolism and steroidogenesis in H295R cells treated with mitotane and/or rosuvastatin. Gene expression was measured by RT-qPCR. H295R cells were treated for $48 \mathrm{~h}$ with mitotane (25 or 50 $\mu \mathrm{M}$; M25, M50), rosuvastatin (50 $\mu \mathrm{M}$; R50), alone or in combination. (A) 3-Hydroxy-3-methylglutaryl-CoA reductase (HMGCR) gene expression. (B) ATP-binding cassette transporter (ABCA1) gene expression. (C) Steroidogenic acute regulatory protein $(S t A R)$ gene expression. (D) Cytochrome P450, family 11, subfamily A, polypeptide 1 (CYP11A1) gene expression. Results are the means \pm SEM of 8 independent determinations from 2 different experiments. Statistical analysis was carried out with the non-parametric Kruskal-Wallis multiple comparison tests followed by a Dunn's post hoc test. ${ }^{* *} \mathrm{P}<0.01$ and ${ }^{* * * *} \mathrm{P}<0.001 \mathrm{vs}$. DMSO condition; ${ }^{\circledR} \mathrm{P}<0.05,{ }^{\S \S} \mathrm{P}<0.01$ and ${ }^{\S \S \S} \mathrm{P}<0.001$ vs. mitotane condition; ns, not significant.
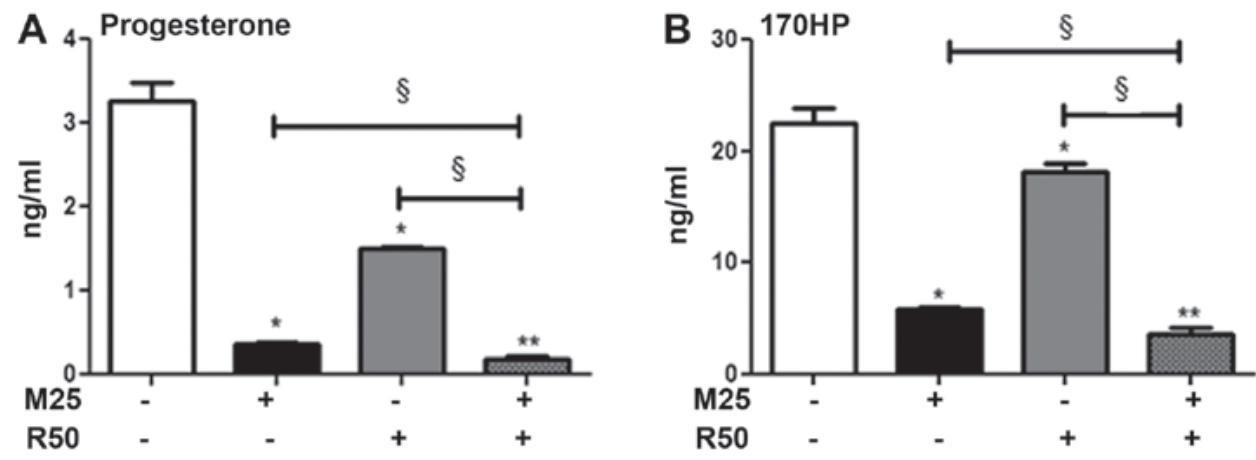

Figure 4. Steroidogenesis in H295R cells treated with mitotane and/or rosuvastatin. (A) Progesterone; (B) 17-hydroxyprogesterone (17OHP). H295R cells were treated for $48 \mathrm{~h}$ with mitotane $(25 \mu \mathrm{M}$; M25), rosuvastatin $(50 \mu \mathrm{M} ; \mathrm{R} 50)$ alone or in combination. Progesterone and $17 \mathrm{OHP}$ concentrations in cell supernatants were measured by LC-MS/MS. Results are the means \pm SEM of 4 independent determinations. Statistical analysis was carried out with the non-parametric Kruskal-Wallis multiple comparison tests followed by a Dunn's post hoc test. ${ }^{*} \mathrm{P}<0.05$ and ${ }^{* *} \mathrm{P}<0.001$ vs. DMSO condition; ${ }^{\circledR} \mathrm{P}<0.05$ vs. mitotane condition.

no direct effect on the activity of HMGCR at least in vitro. The lack of a direct effect of mitotane in vitro associated with the difficulties to accurately evaluate HMGCR activity (data not shown) in the cells did not prompt us to examine the activity in H295R cells under various experimental settings.

Effect of mitotane and rosuvastatin on cholesterol metabolism-related gene expression. We then examined the expression of several genes involved in cholesterol metabolism, including the HMGCR gene, encoding a key player in the intracellular free cholesterol balance, and the $A B C A 1$ gene, encoding a protein that allows cholesterol efflux from the cell.

Rosuvastatin (50 $\mu \mathrm{M}$; R50) alone did not exert any effect, but acted in combination with mitotane at $50 \mu \mathrm{M}$ to significantly reduce $H M G C R$ expression (Fig. 3A). Rosuvastatin $(50 \mu \mathrm{M}$; R50) significantly reduced $A B C A 1$ gene expression, alone or in combination with mitotane 25 and $50 \mu \mathrm{M}$ (Fig. 3B). However, rosuvastatin $(50 \mu \mathrm{M})$ did not alter $L D L R$ or $S r B 1$ gene expression, regardless of the duration of treatment, while mitotane inhibited the expression of these genes (data not shown). 

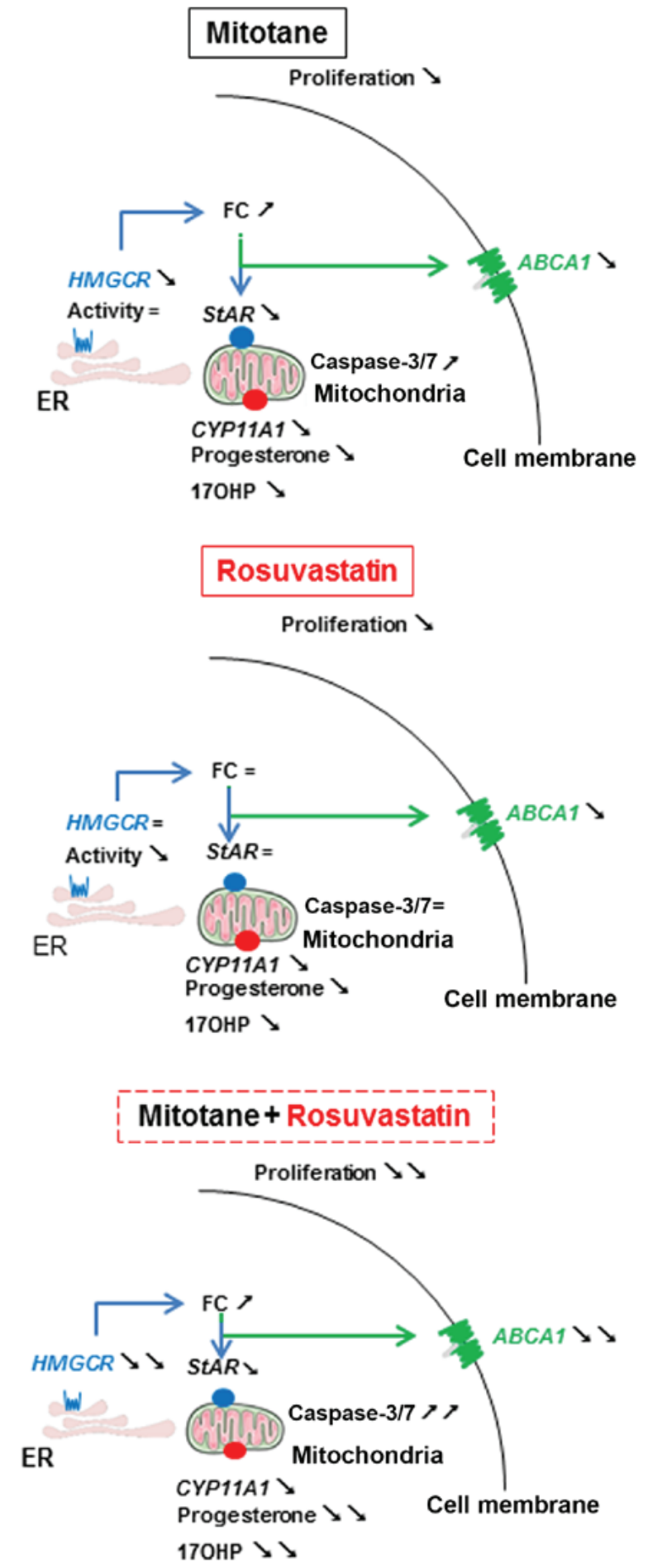

Figure 5. Mechanisms of action of mitotane and rosuvastatin in H295R cells. Arrows facing to the bottom right indicate decreased expression, concentration or activity compared to DMSO. Arrows facing to the top and right indicate an increased expression, concentration or activity compared to DMSO. The '=' symbol indicates the same expression, concentration or activity compared to DMSO. FC, free cholesterol; LDL-R, LDL receptor; SrB1, scavenger receptor $\mathrm{B} 1$; ABCA1, ATP-binding cassette transporter; ER, endoplasmic reticulum; HMGCR or HMGCoA reductase: 3-hydroxy-3-methyl-glutaryl-coenzyme A reductase; StAR, steroidogenic acute regulatory protein; CYP11A1, cytochrome P450, family 11, subfamily A, polypeptide $1 ; 17 \mathrm{OHP}$, 17 hydroxyprogesterone.

Effect of mitotane and rosuvastatin on steroidogenesis. We then examined the expression of genes involved in steroidogenesis, including $S t A R$, encoding the cholesterol transporter facilitating transfer to the mitochondria, and CYP11A1, encoding the first limiting step of steroid synthesis catalyzing cholesterol to pregnenolone (Fig. 3C and D). The expression of StAR was significantly reduced by mitotane in a concentration- (25 and $50 \mu \mathrm{M}$; M25 and M50). Rosuvastatin (50 $\mu \mathrm{M}$; R50) did not exert any significant effect when used alone, but slightly prevented the mitotane-induced reduction in StAR expression. With respect to CYP11A1 expression, mitotane alone significantly reduced its expression (25 and $50 \mu \mathrm{M}$; M25 and M50) (Fig. 3D). Rosuvastatin (50 $\mu \mathrm{M} ; \mathrm{R} 50$ ) alone significantly reduced the expression of CYP11A1.

We then evaluated the steroid-secreting capacities of the H295R cells (Fig. 4A). As previously demonstrated $(2,11)$, mitotane (25 $\mu \mathrm{M} ; \mathrm{M} 25)$ alone decreased the concentration of cortisol and corticosterone in the supernatants of H295R cells following $48 \mathrm{~h}$ of treatment (Fig. S3). Rosuvastatin (50 $\mu \mathrm{M}$; R50) alone decreased the progesterone and 17OHP concentrations. The combination of mitotane $25 \mu \mathrm{M}$ and rosuvastatin $50 \mu \mathrm{M}$ exhibited a significant potentiation effect in inhibiting steroidogenesis, with progesterone secretion reduced by $47 \%$ (Fig. 4A) and that of $17 \mathrm{OHP}$ reduced by $37 \%$ (Fig. 4B).

A visual summary of the mechanisms of action of mitotane and rosuvastatin in the H295R cells described in this study is presented in Fig. 5.

\section{Discussion}

The objective of this study was to better understand the effects of mitotane and rosuvastatin in H295R human adrenocortical carcinoma cells. The main results are summarized in Fig. 5. In this study, we confirmed that mitotane induced apoptosis, reduced cell viability and inhibited steroidogenesis $(13,14)$ We also confirmed that mitotane induced an increase in the intracellular free cholesterol concentration, as recently described by Sbiera et al (5). To gain further insight into the mechanisms involved, we examined the effect of mitotane on the expression of genes involved in cholesterol metabolism. Mitotane significantly decreased the expression of genes involved in the cellular intake of exogenous cholesterol, such as $L D L R$ and $\operatorname{SrBl}$ (data not shown), but also in de novo cholesterol synthesis (HMGCR). On the other hand, mitotane reduces the expression of $A B C A 1$, which is involved in the cellular efflux of cholesterol (14) and inhibits SOAT1 (5), which esterifies free cholesterol. One limitation of this study was that no western blot analysis was carried out to confirm/complete our findings. The observed increase in the intracellular free cholesterol concentration shows the predominance of the effects of the latter over those leading to decrease the cholesterol concentration. These observations are reminiscent of the mechanisms of action of ATR-101, a potent inhibitor of SOAT1 (15) and ABCA1 (14) and currently under clinical development (phase II) for the treatment of adrenocortical carcinoma (Atterocor Inc., Ann Arbor, MI, USA) (16). We hypothesized that mitotane induces MAM dysfunction (4), together with a decrease in steroidogenesis via CYP11A1 inhibition, an increase in free cholesterol inducing ER stress via TSPO, as well as SOAT1 inhibition and an increase in intramitochondrial calcium responsible for apoptosis $(2,13)$.

Statins might play a relevant role in oncology as they induce antiproliferative effects in vivo (9). For the first time in this study, at least to the best of our knowledge, we 
examined the effect of rosuvastatin in ACC cells. Rosuvastatin alone reduced cell viability at high concentrations, without inducing apoptosis, nor altering intracellular free cholesterol. Other cell lines in vitro (breast and glioblastoma cells) have been used to demonstrate the anti-proliferative properties of rosuvastatin at similar concentrations $\left(\mathrm{IC}_{50}\right.$ between 18 and $75 \mu \mathrm{M}$ rosuvastatin) (17). The lack of an effect of statins on intracellular free cholesterol has already been shown in H295R cells with simvastatin (18), whereas a decrease of HMGCoA reductase activity would be expected. Thus, we may assume that the role of the HMGCoA reductase pathway is negligible in adrenocortical cells.

In this study, we demonstrated that in vitro, rosuvastatin potentiated the effects of mitotane by increasing apoptosis and decreasing cell viability. However, the underlying mechanisms remain unknown. Alternate mechanisms, such as autophagy or necroptosis could be involved and thus further investigations are required into this issue. Partial potentiation also occurred for the inhibition of the expression of $H M G C R$ and $A B C A 1$, but no effect was observed on $L D L R$ and $S r B 1$ expression (data not shown) nor on intracellular free cholesterol. Taken together, these observations confirm a potentiation effect of rosuvastatin on mitotane action at the cellular level explained by the inhibition of genes involved in cholesterol metabolism, while no argument supports an influence of rosuvastatin on either mitotane capture or efflux. We have previously demonstrated that a mitochondrial uptake of mitotane significantly increased when cells are cultured with BLT1, an $\mathrm{SrB1}$ receptor inhibitor suggesting an involvement of $\mathrm{SrB} 1$ in mitotane efflux (3). However, in the present study, no effect on mitochondrial mitotane concentrations was observed when the cells were exposed to both mitotane and rosuvastatin.

Statins are already prescribed in clinical practice for the treatment of mitotane-induced dyslipidemia (6). Such dyslipidemia reduces the efficacy of mitotane (3) and overestimates plasma mitotane level measurements (7). This study demonstrated that rosuvastatin also had a direct effect at the cellular level. Statins could therefore be used for their dual action on mitotane transport and bioavailability by reducing lipoprotein concentrations, thus facilitating mitotane efficacy in vivo (3) as well as potentiating cellular action of mitotane. However, not all statins are suitable for such a combined treatment, given that mitotane activates hepatic cytochrome CYP3A4, statins that are not metabolized by this cytochrome seem to be more appropriate for combined therapy (12). Based on the findings of this study, rosuvastatin seems to be a good candidate given its potentiating action with mitotane in vitro. Further prospective studies are warranted to explore the potential benefits of combining mitotane and statins in patients with ACC treated with mitotane.

To conclude, this study demonstrates a potentiating action of mitotane and rosuvastatin in H295R cells. The clinical benefit of this combination remains to be validated in patients and, if confirmed, should lead to a better management of patients with ACC.

\section{Acknowledgements}

GB was a recipient of an HRA PHARMA/SFE (Société Française d'Endocrinologie) fellowship.

\section{Funding}

This study was supported in part by grants from Institut National de la Santé et de la Recherche Médicale (Inserm) and Université Paris-Sud.

\section{Availability of data and materials}

The datasets used and/or analyzed during the current study are available from the corresponding author on reasonable request.

\section{Authors' contributions}

GB performed and analyzed cell viability, apoptosis analysis, intracellular free cholesterol measurements, RT-qPCR experiments. LA and AN performed and analyzed PARP expression by western blotting. AS and AP performed and analyzed mitotane measurements. AL performed and analyzed HMGCoA reductase activity. EP performed the LC-MS/MS experiments. $\mathrm{EB}, \mathrm{SH}$ and ML designed the study. GB, ML and SH interpreted the data, and were major contributors to the writing the manuscript. EB contributed to the drafting of the manuscript. All authors have read and approved the version to be published and approved its submission.

\section{Ethics approval and consent to participate}

Not applicable.

\section{Patient consent for publication}

Not applicable.

\section{Competing interests}

The authors state that they have no competing interests.

\section{References}

1. Berruti A, Baudin E, Gelderblom H, Haak HR, Porpiglia F, Fassnacht $\mathrm{M}$ and Pentheroudakis G; ESMO Guidelines Working Group: Adrenal cancer: ESMO Clinical Practice Guidelines for diagnosis, treatment and follow-up. Ann Oncol 23 (Suppl 7): vii131-vii138, 2012.

2. Hescot S, Slama A, Lombès A, Paci A, Remy H, Leboulleux S, Chadarevian R, Trabado S, Amazit L, Young J, et al: Mitotane alters mitochondrial respiratory chain activity by inducing cytochrome $c$ oxidase defect in human adrenocortical cells. Endocr Relat Cancer 20: 371-381, 2013.

3. Hescot S, Seck A, Guerin M, Cockenpot F, Huby T, Broutin S, Young J, Paci A, Baudin E and Lombès M: Lipoprotein-free mitotane exerts high cytotoxic activity in adrenocortical carcinoma. J Clin Endocrinol Metab 100: 2890-2898, 2015.

4. Hescot S, Amazit L, Lhomme M, Travers S, DuBow A, Battini S, Boulate G, Namer IJ, Lombes A, Kontush A, et al: Identifying mitotane-induced mitochondria-associated membranes dysfunctions: Metabolomic and lipidomic approaches. Oncotarget 8: 109924-109940, 2017.

5. Sbiera S, Leich E, Liebisch G, Sbiera I, Schirbel A, Wiemer L, Matysik S, Eckhardt C, Gardill F, Gehl A, et al: Mitotane inhibits sterol-O-acyl transferase 1 triggering lipid-mediated endoplasmic reticulum stress and apoptosis in adrenocortical carcinoma cells. Endocrinology 156: 3895-3908, 2015.

6. Shawa H, Deniz F, Bazerbashi H, Hernandez M, VassilopoulouSellin R, Jimenez C and Habra MA: Mitotane-induced hyperlipidemia: A retrospective cohort study. Int J Endocrinol 2013: 624962, 2013. 
7. Paci A,Hescot S, Seck A, Jublanc C, Mercier L, Vezzosi D, Drui D, Quinkler M, Fassnacht M, Bruckert E, et al: Dyslipidemia causes overestimation of plasma mitotane measurements. Endocrinol Diabetes Metab Case Rep 2016: 150135, 2016.

8. Eisenhauer EA, Therasse P, Bogaerts J, Schwartz LH, Sargent D, Ford R, Dancey J, Arbuck S, Gwyther S, Mooney M, et al: New response evaluation criteria in solid tumours: Revised RECIST guideline (version 1.1). Eur J Cancer 45: 228-247, 2009.

9. Pisanti S, Picardi P, Ciaglia E, D'Alessandro A and Bifulco M Novel prospects of statins as therapeutic agents in cancer. Pharmacol Res 88: 84-98, 2014.

10. Clendening JW, Pandyra A, Boutros PC, El Ghamrasni S Khosravi F, Trentin GA, Martirosyan A, Hakem A, Hakem R, Jurisica I, et al: Dysregulation of the mevalonate pathway promotes transformation. Proc Natl Acad Sci USA 107: 15051-15056, 2010.

11. Hescot S, Paci A, Seck A, Slama A, Viengchareun S, Trabado S, Brailly-Tabard S, Al Ghuzlan A, Young J, Baudin E, et al: The lack of antitumor effects of o,p'DDA excludes its role as an active metabolite of mitotane for adrenocortical carcinoma treatment. Horm Cancer 5: 312-323, 2014.

12. Takeshita A, Igarashi-Migitaka J, Koibuchi N and Takeuchi Y: Mitotane induces CYP3A4 expression via activation of the steroid and xenobiotic receptor. J Endocrinol 216: 297-305, 2013.

13. Touitou Y, Moolenaar AJ, Bogdan A, Auzéby A and Luton JP o,p'-DDD (mitotane) treatment for Cushing's syndrome: Adrenal drug concentration and inhibition in vitro of steroid synthesis. Eur J Clin Pharmacol 29: 483-487, 1985.
14. Burns VE and Kerppola TK: ATR-101 inhibits cholesterol efflux and cortisol secretion by ATP-binding cassette transporters, causing cytotoxic cholesterol accumulation in adrenocortical carcinoma cells. Br J Pharmacol 174: 3315-3332, 2017.

15. LaPensee CR, Mann JE, Rainey WE, Crudo V, Hunt SW III and Hammer GD: ATR-101, a selective and potent inhibitor of Acyl-CoA acyltransferase 1, induces apoptosis in H295R adrenocortical cells and in the adrenal cortex of dogs. Endocrinology 157: 1775-1788, 2016.

16. U.S. National Library of Medicine: A Study of ATR-101 for the Treatment of Endogenous Cushing's Syndrome. Identification No. NCT03053271. https://clinicaltrials. gov/ct2/show/NCT03053271. Accessed February 15, 2019.

17. Jiang P, Mukthavaram R, Chao Y, Nomura N, Bharati IS, Fogal V, Pastorino S, Teng D, Cong X, Pingle SC, et al: In vitro and in vivo anticancer effects of mevalonate pathway modulation on human cancer cells. Br J Cancer 111: 1562-1571, 2014.

18. Guldvang A, Hansen CH, Weisser JJ, Halling-Sørensen B and Styrishave B: Simvastatin decreases steroid production in the H295R cell line and decreases steroids and FSH in female rats. Reprod Toxicol 58: 174-183, 2015. 